\title{
Clinical impact of neurocognitive deficits after cardiac surgery
}

\author{
Hadi D. Toeg, MD, ${ }^{\mathrm{a}}$ Howard Nathan, MD, ${ }^{\mathrm{b}}$ Fraser Rubens, MD, MSc, ${ }^{\mathrm{a}}$ Denise Wozny, BA, ${ }^{\mathrm{b}}$ and \\ Munir Boodhwani, MD, MMSc ${ }^{\mathrm{a}}$
}

Objectives: Postoperative neurocognitive deficits (POCDs) have been found to occur frequently after cardiac surgery. Although POCDs have received significant attention in the medical literature and public media, the true clinical impact of these deficits on patient outcomes and quality of life (QOL) is not well defined.

\begin{abstract}
Methods: Neuropsychometric testing was performed on 696 patients undergoing coronary artery bypass surgery using a battery of tests divided into 4 domains; memory, attention, speed, and psychomotor function. These were performed preoperatively, at hospital discharge, and at 3 months postoperatively. POCDs were defined as a drop in scores by 1 standard deviation in 1 domain or more. QOL was assessed using Short Form 36 and clinical outcomes were recorded.
\end{abstract}

Results: POCDs were identified in $265(38 \%)$ patients at discharge and in $132(19 \%)$ at 3 months. There was no observed difference in mortality or major morbidity in patients with or without POCDs. Predictors of POCDs at discharge were elevated preoperative creatinine $(P=.04)$, increased cardiopulmonary bypass time $(P=.005)$, and diabetes $(P=.003)$. At 3 months, patients had improvements in both physical and mental components of QOL, independent of the occurrence of POCDs $(P>.5)$. Independent predictors of improved QOL included younger age, severe preoperative anginal symptoms, normal left ventricular function, absence of postoperative wound infection, but not POCDs.

Conclusions: Neurocognitive deficits can be frequently detected on comprehensive neuropsychometric testing after cardiac surgery. However, they are not associated with any clinically important differences in patient outcome or in QOL after surgery. (J Thorac Cardiovasc Surg 2013;145:1545-9)

Earn CME credits at

http://cme.ctsnetjournals.org

Postoperative neurocognitive deficits (POCDs) have been reported to occur in $30 \%$ to $80 \%$ of patients after cardiac surgery. ${ }^{1}$ POCDs are subtle changes in higher cognitive function that can be identified using comprehensive neuropsychiatric testing assessing a variety of neurocognitive domains like memory, attention, speed, and psychomotor function. Recent studies have identified POCDs in other populations including patients undergoing major noncardiac surgical procedures as well as those undergoing coronary angiography. ${ }^{2,3}$ POCDs have received significant attention in the medical literature and in the public media

\footnotetext{
From the Departments of Cardiac Surgery ${ }^{\mathrm{a}}$ and Anesthesiology, ${ }^{\mathrm{b}}$ The Ottawa Hospital, University of Ottawa, Ottawa, Ontario, Canada.

Funded by the Canadian Institutes for Health Research.

Disclosures: Authors have nothing to disclose with regard to commercial support.

Read at the 92nd Annual Meeting of The American Association for Thoracic Surgery, San Francisco, California, April 28-May 2, 2012.

Received for publication Dec 23, 2012; revisions received Feb 12, 2013; accepted for publication Feb 27, 2013; available ahead of print March 26, 2013.

Address for reprints: Munir Boodhwani, MD, MMSc, University of Ottawa Heart Institute, 40 Ruskin St, Ottawa, Ontario, Canada, K1Y 4W7 (E-mail: mboodhwani@ottawaheart.ca).

$0022-5223 / \$ 36.00$

Copyright (c) 2013 by The American Association for Thoracic Surgery http://dx.doi.org/10.1016/j.jtcvs.2013.02.061
}

because of the suggestion that they may contribute to a delay in postoperative recovery, delayed return to work, and impaired quality of life (QOL). ${ }^{4}$ Although the incidence of POCDs in patients undergoing coronary artery bypass graft (CABG) surgery can be high, the majority of patients tend to return to their preoperative baseline neurocognitive status. ${ }^{5}$ Studies have also demonstrated that patients with early POCDs are at greater risk for long-term neurocognitive decline at 5 years. ${ }^{6-8}$

The mechanisms of POCDs continue to be debated and are likely multifactorial. Predictors of the occurrence of POCDs include underlying cerebrovascular disease, advanced age, length of cardiopulmonary bypass (CPB) time, manipulation of the ascending aorta, and cerebral hyperthermia. ${ }^{9,10}$ Other less consistently demonstrated predictors of POCDs include hypertension, diabetes, dementia, peripheral vascular disease, and lower educational level. ${ }^{10,11}$

Most studies look for predictors and methods of reducing POCDs, but no study has thoroughly investigated the true clinical impact of these deficits. ${ }^{1,6,9,12}$ When QOL was measured in patients having POCDs, QOL and productive working status were both significantly reduced compared with those in patients without POCDs. Conversely, most studies investigating the impact of change in QOL after CABG surgery revealed overall improvements in QOL. ${ }^{13,14}$ Given that this study did not measure preoperative QOL and thus did not calculate the change in QOL, attributing reduced QOL to POCDs cannot be fully concluded. ${ }^{12}$ 


\section{Abbreviations and Acronyms \\ $\mathrm{CABG}=$ coronary artery bypass graft \\ $\mathrm{CPB}=$ cardiopulmonary bypass \\ ICU = intensive care unit \\ POCD = postoperative neurocognitive deficit \\ QOL $\quad=$ quality of life \\ SF-12 = Short Form Health Survey-12 \\ (or -36) (or -36)}

This study aimed to delineate the true clinical impact of POCDs in a large cohort of patients undergoing on-pump CABG surgery. A variety of variables assessing mortality, morbidity, and QOL were assessed in all patients and their relationship to POCDs was examined.

\section{PATIENTS AND METHODS \\ Patient Population}

The study population consisted of 696 patients over the age of 60 years who had been accepted for nonurgent CABG surgery at the University of Ottawa Heart Institute between 1995 and 2004. This cohort of patients had been previously enrolled in 3 separate randomized trials evaluating the effects of intraoperative temperature and cell salvage on POCDs. ${ }^{15-17}$ Further details were reviewed by Boodhwani and associates ${ }^{9}$ in 2006. The study protocols were approved by the Institutional Review Board of the University of Ottawa Heart Institute and written informed consent was obtained from all subjects.

Patients included in the trial were more than the age of 60 years, fluent in either English or French, and undergoing nonemergency CABG surgery. Patients were excluded if they had a preoperative neurologic deficit of any etiology, Parkinson disease, physical disability preventing neuropsychometric testing, Mini-Mental Status Exam score less than 24, on intravenous nitroglycerin, documented coagulopathy (or international normalized ratio $>1.3$ ), significant renal dysfunction (serum creatinine $>2$ times nor$\mathrm{mal})$, and preoperative atrial fibrillation.

\section{Intraoperative Protocol}

The protocol for the various study-related interventions has been previously described. ${ }^{9,15,16,18}$ All procedures were performed through a median sternotomy approach using CPB instituted via cannulation of the ascending aorta and a 2-stage right atrial cannulation along with a membrane oxygenator and a $43-\mu \mathrm{m}$ arterial line filter (Cobe Cardiovascular, Arvada, Colo), with a nonpulsatile flow at 2.5 to $2.8 \mathrm{~L} / \mathrm{min} / \mathrm{m}^{2}$, and without the use of left ventricular vents. Mean arterial pressure was maintained between 50 and $80 \mathrm{~mm} \mathrm{Hg}$ using phenylephrine or isoflurane. After the aortic crossclamp was applied, cardiac arrest was initiated and maintained with antegrade cold blood cardioplegia. Proximal anastomoses were performed either using a side-biting clamp on the aorta or while on CPB. Tranexamic acid was given to patients to reduce blood loss. Standardized protocols were used for postoperative care.

\section{Neuropsychometric Evaluation}

Testing was conducted in accordance with the consensus statement on neurobehavioral evaluation after cardiac surgery. ${ }^{19} \mathrm{~A}$ battery of 14 tests divided into 4 domains assessing memory, attention, speed, and psychomotor function were performed on patients preoperatively, at hospital discharge, and at 3 months' follow-up. Learning efficiency and memory consolidation were evaluated with a verbal list learning procedure (Buschke Selective Reminding administration and scoring or Rey Auditory and Verbal Learning Test). Attention span was evaluated with the Wechsler Adult Intelligence Scale-Revised Digit Span. Speed and motor domains were assessed by Finger Tapping Test, and Letter and Category Fluency was used to assess speed and flexibility of verbal thought processes. Psychomotor function was measured by Trails A and B, Grooved Pegboard, and Symbol Digit Modalities Test (oral administration). Neurocognitive testing was performed at all time points under similar conditions and, whenever possible, by the same evaluator. Alternate forms were used to reduce learning effects. To optimize reliability of the neuropsychometric evaluation, we had the psychometrists trained and periodically audited by the same neuropsychologist. All patients were ambulating and fit for discharge at the time of postoperative neuropsychometric testing.

QOL was measured either by the Short Form Health Survey-36 $(\mathrm{SF}-36)^{20}$ or the equivalent condensed Short Form Health Survey-12 $(\mathrm{SF}-12) .{ }^{21}$ The total battery of tests required approximately 60 minutes to administer.

\section{Statistical Analysis}

Neuropsychometric results were analyzed both as continuous and dichotomous outcomes. Individual psychometric test scores yielded continuous measures repeated at 3 time points including preoperative, at hospital discharge, and at 3 months' follow-up.

For categorical analysis, the neuropsychometric tests were combined into 4 cognitive domains using factor analysis with orthogonal rotation as detailed by Newman and coworkers. ${ }^{6}$ This reduced the individual test scores into 4 factors that were uncorrelated and accounted for $80 \%$ of the variance present in the test battery corresponding to the following areas of cognitive functioning: verbal memory, attention, motor function, and psychomotor speed and dexterity. The scores were adjusted so that an increase in score always indicated better performance, and a composite score was formed by adding the 4 factors (composite cognitive index). A patient was deemed to have had a POCD if 1 or more factor scores decreased by at least 1 standard deviation. A secondary analysis was also performed using reliable change index methodology to account for practice effects. A cohort of 75 nonsurgical control subjects with coronary artery disease was tested at the same intervals, and this information was used to adjust for practice learning effects in the study patients as described by Rasmussen and colleagues. ${ }^{22}$

QOL data, obtained from the SF-12 or SF-36 questionnaires, were analyzed separately for the physical and mental domains. QOL scores were normalized using the preoperative (baseline) scores, and percent change at 3 months was calculated for all patients. Logistic and linear regression models were constructed to examine predictors of POCDs and of QOL, respectively.

Data are presented as mean \pm standard deviation or median (interquartile range). Odds ratios are presented with their $95 \%$ confidence intervals. Continuous variables were compared by using unpaired $t$ tests if normally distributed or the Wilcoxon rank-sum test for nonnormally distributed continuous variables. Categorical data were compared using $\chi^{2}$ or Fisher exact tests. A liberal threshold of $P<.20$ was used to select variables for the construction of the multivariable logistic regression model. This model was constructed by first choosing the best single variable model as determined by the likelihood ratio test. The next covariate to be added was identified by considering all possible 2-variable models and selecting the one with the greatest likelihood. New variables were then added to the model until the addition of covariates no longer significantly improved the model. All the significant univariate predictors that did not enter the final model were individually added to the final model to assess confounding. The linearity assumption for continuous predictors was evaluated by dividing the variable into equal width bins and examining the adjusted odds ratio across the bins. Finally, the model was boot-strapped 1000 times to assess whether the model was overfit. All analyses were conducted in SAS version 9.1 (SAS Institute, Inc, Cary, NC). 
TABLE 1. Preoperative and intraoperative variables in patients with or without POCDs demonstrating no significant differences (expressed either as mean or percentage \pm standard error of the mean)

\begin{tabular}{lccc}
\hline \multicolumn{1}{c}{ Variable } & $\begin{array}{c}\text { No POCD } \\
(\mathbf{n = 5 3 1 )}\end{array}$ & $\begin{array}{c}\text { POCD } \\
(\mathbf{n}=\mathbf{1 2 1})\end{array}$ & $\begin{array}{c}\boldsymbol{P} \\
\text { value }\end{array}$ \\
\hline Age & $64 \pm 9$ & $66 \pm 9$ & .19 \\
Hypertension & $268(67 \%)$ & $67(74 \%)$ & .14 \\
Diabetes & $162(31 \%)$ & $42(35 \%)$ & .44 \\
LV dysfunction & $183(34 \%)$ & $40(33 \%)$ & .76 \\
Severe angina (class III/IV) & $356(67 \%)$ & $77(64 \%)$ & .47 \\
Male gender & $470(89 \%)$ & $106(88 \%)$ & .79 \\
BMI & $29.1 \pm 4.5$ & $29.2 \pm 4.9$ & .76 \\
Serum creatinine & $92 \pm 18$ & $91 \pm 20$ & .73 \\
Hematocrit & $41 \pm 4$ & $42 \pm 4$ & .10 \\
Carotid disease & $19(5.7 \%)$ & $2(2.5 \%)$ & .39 \\
Peripheral vascular disease & $41(12 \%)$ & $13(16 \%)$ & .36 \\
CPB time & $86 \pm 28$ & $82 \pm 25$ & .12 \\
No. of bypass grafts & $3.1 \pm 0.7$ & $3.1 \pm 0.7$ & .72 \\
\hline
\end{tabular}

$P O C D$, Postoperative neurocognitive deficit; $L V$, left ventricular; $B M I$, body mass index; $C P B$, cardiopulmonary bypass.

\section{RESULTS}

POCDs were observed in $38 \%$ of patients $(n=265 / 696)$ at discharge from the hospital and $19 \%(n=132 / 696)$ at 3 months' follow-up. In patients aged 75 years or older, the incidence of POCDs at discharge was $43 \%$ whereas the incidence in patients under 75 years of age was $37 \%$ $(P=.29)$. Three months after the operation, patients over the age of 75 had a $25 \%$ incidence of POCDs compared with $18 \%$ in those less than 75 years of age $(P=.15)$.

Of patients in whom POCDs were diagnosed at 3 months, $97 \%(n=117)$ had deficits in just 1 domain and $3 \%(n=4)$ of patients noticed deficits in 2 domains or more. From the $132(19 \%$ of total) patients who had POCDs at 3 months, only $54(41 \%)$ had deficits in the same neuropsychiatric domains as at the time of discharge. Completeness of followup was achieved in $100 \%$ before the operation, $99 \%$ after discharge from the hospital, and $94 \%$ at 3 months. There were no significant differences noted in preoperative and intraoperative variables in patients with or without POCDs (Table 1).

The overall in-hospital mortality rate was $11(1.6 \%)$ patients and the mortality rate of survivors at 3 months was 2 $(0.3 \%)$. Postoperative outcomes comparing patients with POCDs and those without are illustrated in Table 2. Patients with POCDs had longer intensive care unit (ICU) stays $(P=.03)$, and twice the number of patients with POCDs had a prolonged ICU stay ( $>48$ hours; $P=.01$ ). The overall hospital length of stay was slightly but not significantly greater in patients with POCDs $(P=.11)$. The incidence of other postoperative outcomes including myocardial infarction, reopening for bleeding, surgical site infection, and length of ventilation were similar between patients with or without POCDs (Table 2).
TABLE 2. Postoperative variables in patients with or without POCDs (expressed either as mean or percentage \pm standard error of the mean)

\begin{tabular}{lccc}
\hline \multicolumn{1}{c}{ Variable } & $\begin{array}{c}\text { No POCD } \\
(\mathbf{n}=\mathbf{5 3 1})\end{array}$ & $\begin{array}{c}\text { POCD } \\
(\mathbf{n}=\mathbf{1 2 1})\end{array}$ & $\begin{array}{c}\boldsymbol{P} \\
\text { value }\end{array}$ \\
\hline Three-month mortality & $2(0.4 \%)$ & 0 & .66 \\
Postop MI & $9(1.7 \%)$ & 0 & .22 \\
Reopening for bleeding & $20(3.7 \%)$ & $6(4.9 \%)$ & .68 \\
Prolonged ICU stay (>48 h) & $66(12 \%)$ & $26(21 \%)$ & .01 \\
ICU LOS & $1.3 \pm 1.3$ & $1.8 \pm 2.9$ & .03 \\
Hospital LOS & $6.4 \pm 3.0$ & $6.8 \pm 5.3$ & .11 \\
Surgical site infection & $12(2.3 \%)$ & $3(2.5 \%)$ & .74 \\
Length of ventilation (h) & $12 \pm 18$ & $16 \pm 41$ & .19 \\
\hline POCDs, Postoperative neurocognitive deficits; $M I$, myocardial infarction; $I C U$, \\
intensive care unit; $L O S$, length of stay.
\end{tabular}

\section{Neurocognitive Outcomes}

POCDs were defined as a reduction by at least 1 standard deviation in more than 1 domain of interest. Predictors of POCDs at discharge and at 3 months postoperatively were determined by multivariate regression analysis (Table 3 ). Predictors of POCDs at discharge from the hospital were diabetes $(P<.0001)$, increased preoperative creatinine level $(P=.003)$, and longer CPB time $(P=.008)$. Three months after the operation, the only independent predictor for POCDs was prolonged ICU stay ( $>48$ hours; $P=.01$ ). Inasmuch as this study compiled data from 3 randomized controlled trials, the treatment conditions in each study were added into the multivariable model demonstrating that these interventional conditions were not significant predictors of POCDs.

\section{QOL Assessment}

Baseline QOL was assessed preoperatively and was compared with QOL 3 months after the operation by administrating the SF-36 or the SF-12 test. At 3 months, patients had significant improvements in both physical $(34 \% \pm 2 \%$ increase from baseline) and mental (10\% $\pm 1 \%$ increase from baseline) components of QOL independent of the occurrence of POCDs $(P>.5)$. Using a multivariable regression model, independent predictors of improved QOL were determined and depicted in Table 4. The strongest predictors of improved QOL included younger age $(P<.001)$, severe preoperative anginal class $(P<.001)$, absence of postoperative sternal wound infection $(P=.03)$, and normal

TABLE 3. Multivariate regression analysis model demonstrating predictors of POCDs at discharge and 3 months after CABG surgery

\begin{tabular}{lccc}
\hline \multicolumn{1}{c}{ Variable } & Odds ratio & $\mathbf{9 5} \% \mathbf{C I}$ & $\boldsymbol{P}$ value \\
\hline \multirow{4}{*}{ At discharge } & & \\
Diabetes & 1.95 & $1.39-2.73$ & $<.0001$ \\
Preop creatinine & 1.02 & $1.01-1.03$ & $<.003$ \\
CPB time & 1.01 & $1.00-1.02$ & .008 \\
& At 3 months & & \\
Prolonged ICU stay & 1.92 & $1.16-3.19$ & .01 \\
\hline
\end{tabular}

$P O C D s$, Postoperative neurocognitive deficits; $C A B G$, coronary artery bypass graft $C I$, confidence interval; $C P B$, cardiopulmonary bypass; $I C U$, intensive care unit. 
TABLE 4. Predictors of improved QOL in patients undergoing surgery

\begin{tabular}{lccc}
\hline \multicolumn{1}{c}{ Variable } & Odds ratio & $\mathbf{9 5} \%$ CI & $\boldsymbol{P}$ value \\
\hline Younger age & 4.00 & $2.43-7.14$ & $<.001$ \\
Severe CCS angina class & 3.29 & $1.9-4.67$ & $<.001$ \\
Normal LV ejection fraction & 1.31 & $1.01-2.6$ & .04 \\
Absence of sternal wound infection & 9.65 & $1.2-18.1$ & .03 \\
Absence of neurocognitive deficit & 0.86 & $0.05-14.9$ & .92 \\
\hline$C I$, Confidence interval; $C C S$, canadian cardiovascular society; $L V$, left ventricular.
\end{tabular}

left ventricular ejection fraction $(P=.04)$; however, the absence of POCDs was not a predictor of improved QOL $(P=.92)$ at 3 months after CABG.

\section{DISCUSSION}

In this large, single-center cohort of on-pump CABG patients undergoing comprehensive neuropsychometric evaluation, we sought to determine the clinical impact of POCDs. We found that the incidence of POCDs in this study was $38 \%$ at discharge followed by an expected decline to $19 \%$ at 3 months after CABG. Furthermore, elderly patients, those older than 75 years of age, had similar incidences of POCDs when compared with younger patients. Predictors of POCDs at discharge included longer CPB time, higher preoperative creatinine level, and diabetes; at 3 months, only prolonged ICU stay was associated with POCDs. The occurrence of POCDs was not associated with increased morbidity or increased hospital stay. Importantly, we found that although patients had significant improvements in physical and mental domains of QOL after surgery, these improvements were independent of the occurrence of POCDs. In summary, this study demonstrates that in patients undergoing CABG surgery, POCDs do not significantly affect clinical outcomes including morbidity, mortality, and QOL.

There was no observed clinically significant difference in mortality or morbidity in patients with or without POCDs in this study. Patients who had POCDs did have statistically significantly longer ICU stay $(P=.03)$ by an average of 0.5 days, along with a trend toward longer hospital stay $(P=.11)$; however, other postoperative complications including length of ventilation, reexploration for bleeding, myocardial infarction, and mortality were similar in both groups. Independent predictors of developing POCDs at discharge included diabetes, increased preoperative creatinine level, and prolonged CPB time, all of which are known risk factors for the development of POCDs. Three months after CABG, only prolonged ICU stay was associated with a higher chance of developing POCDs, whereas the determined predictors of developing POCD at discharge (diabetes, creatinine, CPB) were not present at 3 months. The reasons for the differences may be because of the lower number of patients with POCDs at 3 months or that prolonged ICU stay is more likely to occur in and therefore may be a surrogate marker for patients with a higher burden of comorbidities and more challenging operations.

Postoperative QOL was assessed with either SF-36 or SF12 in all patients undergoing surgery and was compared with their baseline levels. Both components of QOL (mental and physical) were improved from baseline independent of the presence of POCDs $(P>.5)$. Although most patients undergoing $\mathrm{CABG}$ surgery do note an improvement in QOL when compared with baseline, ${ }^{13,14}$ Newman and colleagues $^{12}$ demonstrated a direct correlation between increased neurocognitive decline at 5 years with decreased QOL and lower general health status. Inasmuch as patients experiencing POCDs and late cognitive decline are likely to have a higher burden of comorbidities and are also likely to have poorer baseline QOL, it is difficult to conclude a causative relationship between POCDs and QOL from this study because baseline QOL scores were not measured. In our study, we compared the change in QOL after surgery in both patients with and without POCDs, and no significant difference was appreciated. Furthermore, we adjusted for other variables that are associated with POCDs that may have been different between groups. Even after multivariate analyses for predictors of increased QOL after CABG, the absence or the presence of POCDs was not linked (odds ratio $=0.86 ; P$ value $=.92$ ). The strongest predictors of increased QOL were found to be younger age, higher Canadian Cardiovascular Society angina class, absence of sternal wound infection, and normal ejection fraction. This finding abrogates the notion that POCDs after CABG lead to a poorer QOL and overall general health status.

This study was aimed at elucidating the clinical impact of POCDs in on-pump CABG patients, and we found that there was no appreciable clinical impact or any effect on QOL. These findings, combined with previously published reports, can help to shed light on the nature, mechanism, and relevance of POCDs. Early studies examining this issue identified POCDs as a frequent occurrence associated with late cognitive decline. Their occurrence was attributed to the use and management of CPB. However, several groups have since demonstrated that there are no differences in early and late POCDs between patients undergoing onpump versus off-pump surgery. ${ }^{23,24}$ Various studies have also examined specific CPB management strategies including temperature and use of cell salvage devices and have yielded inconclusive and mixed results. ${ }^{16-18}$ Recent studies have also demonstrated that when CABG patients are compared with appropriately controlled nonsurgical patients with coronary artery disease, the incidence of POCDs is similar at 1 year, suggesting that the preoperative status and preexisting cerebrovascular disease play a more direct role in developing POCDs than the operation itself. ${ }^{25}$ Furthermore, patients with known coronary artery disease are more prone to cognitive decline after any form of intervention (percutaneous coronary intervention or $\mathrm{CABG}$ ) than are 
control patients with no coronary artery disease. ${ }^{3}$ Last, a systematic review determined that the incidence of early and late POCDs after noncardiac surgery was similar and that various treatment strategies to reduce the number of POCDs had no significant impact on patient outcomes. ${ }^{2}$ Of the 132 patients who had POCDs at 3 months, only $41 \%$ had deficits in the same neuropsychiatric domains seen at discharge. This implies that the majority of patients had deficits develop in new domains between the time of hospital discharge and at 3 months of follow-up, further corroborating the concept that POCDs are not likely to be related to the surgery. Combined with our data, these studies suggest that POCDs may be a marker of underlying cerebrovascular disease that is unmasked owing to the stress of a cardiac surgical procedure. These findings also have implications for clinical trials designed to reduce the occurrence of POCDs.

The limitations to this study include the fact that this is a homogeneous, single-center cohort of relatively low-risk patients undergoing coronary surgery and that the results may not be generalizable to other patients undergoing cardiac surgery. Inasmuch as this study combined patients from 3 prospective randomized controlled trials from our institution, confounding variables created by different trial interventions and allocations could potentially affect the conclusions made. ${ }^{15-17}$ However, all 3 trials, when examined individually, did not show any differences in morbidity, mortality, or QOL. Also, these interventional conditions were applied to the multivariate analysis concluding that treatment allocation was not a significant predictor of POCDs. Another limitation relates to the definition of POCDs. Although there is no universally accepted definition, the criteria used in this study yielded an incidence of POCDs that is similar to that which has been reported previously. Differences in POCD definition could have theoretically led to some alterations in study findings.

\section{CONCLUSIONS}

POCDs occur frequently after cardiac surgery and are likely affected by preoperative, intraoperative, and postoperative factors. POCDs are not associated with increased mortality, morbidity, or longer hospital stay. Patients undergoing surgical coronary revascularization uniformly have improvements in QOL that are not affected by POCD occurrence. Although the pathogenesis of POCDs remains uncertain, it is likely that they are a marker of underlying cerebrovascular disease unmasked during a major operation.

\section{References}

1. Stroobant N, Van Nooten G, Van Belleghem Y, Vingerhoets G. The effect of CABG on neurocognitive functioning. Acta Cardiol. 2010;65:557-64.

2. Newman S, Stygall J, Hirani S, Shaefi S, Maze M. Postoperative cognitive dysfunction after noncardiac surgery: a systematic review. Anesthesiology. 2007; 106:572-90.

3. Rosengart TK, Sweet J, Finnin EB, Wolfe P, Cashy J, Hahn E, et al. Neurocognitive functioning in patients undergoing coronary artery bypass graft surgery or percutaneous coronary intervention: evidence of impairment before intervention compared with normal controls. Ann Thorac Surg. 2005;80:1327-34; discussion 1334-5.

4. McKhann GM, Goldsborough MA, Borowicz LM Jr, Selnes OA, Mellits ED, Enger C, et al. Cognitive outcome after coronary artery bypass: a one-year prospective study. Ann Thorac Surg. 1997;63:510-5.

5. Stygall J, Newman SP, Fitzgerald G, Steed L, Mulligan K, Arrowsmith JE, et al. Cognitive change 5 years after coronary artery bypass surgery. Health Psychol. 2003;22:579-86

6. Newman MF, Kirchner JL, Phillips-Bute B, Gaver V, Grocott H, Jones RH, et al. Longitudinal assessment of neurocognitive function after coronary-artery bypass surgery. N Engl J Med. 2001;344:395-402.

7. Selnes OA, Royall RM, Grega MA, Borowicz LM Jr, Quaskey S, McKhann GM Cognitive changes 5 years after coronary artery bypass grafting: is there evidence of late decline? Arch Neurol. 2001;58:598-604.

8. Zimpfer D, Czerny M, Vogt F, Schuch P, Kramer L, Wolner E, et al. Neurocognitive deficit following coronary artery bypass grafting: a prospective study of surgical patients and nonsurgical controls. Ann Thorac Surg. 2004;78:513-8; discussion 518-9.

9. Boodhwani M, Rubens FD, Wozny D, Rodriguez R, Alsefaou A, Hendry PJ, et al. Predictors of early neurocognitive deficits in low-risk patients undergoing onpump coronary artery bypass surgery. Circulation. 2006;114(1 Suppl):I461-6.

10. Ho PM, Arciniegas DB, Grigsby J, McCarthy M Jr, McDonald GO, Moritz TE, et al. Predictors of cognitive decline following coronary artery bypass graft surgery. Ann Thorac Surg. 2004;77:597-603; discussion 603.

11. Stanley TO, Mackensen GB, Grocott HP, White WD, Blumenthal JA Laskowitz DT, et al. The impact of postoperative atrial fibrillation on neurocognitive outcome after coronary artery bypass graft surgery. Anesth Analg. 2002;94: 290-5, table of contents.

12. Newman MF, Grocott HP, Mathew JP, White WD, Landolfo K, Reves JG, et al Report of the substudy assessing the impact of neurocognitive function on quality of life 5 years after cardiac surgery. Stroke. 2001;32:2874-81.

13. Page SA, Verhoef MJ, Emes CG. Quality of life, bypass surgery and the elderly. Can J Cardiol. 1995;11:777-82.

14. Motallebzadeh R, Bland JM, Markus HS, Kaski JC, Jahangiri M. Health-related quality of life outcome after on-pump versus off-pump coronary artery bypass graft surgery: a prospective randomized study. Ann Thorac Surg. 2006;82:615-9.

15. Boodhwani M, Rubens F, Wozny D, Rodriguez R, Nathan HJ. Effects of sustained mild hypothermia on neurocognitive function after coronary artery bypass surgery: a randomized, double-blind study. J Thorac Cardiovasc Surg. 2007;134: 1443-50; discussion 1451-2.

16. Nathan HJ, Wells GA, Munson JL, Wozny D. Neuroprotective effect of mild hypothermia in patients undergoing coronary artery surgery with cardiopulmonary bypass: a randomized trial. Circulation. 2001;104(12 Suppl 1):I85-91.

17. Rubens FD, Boodhwani M, Mesana T, Wozny D, Wells G, Nathan HJ. Cardiotomy Investigators. The cardiotomy trial: a randomized, double-blind study to assess the effect of processing of shed blood during cardiopulmonary bypass on transfusion and neurocognitive function. Circulation. 2007;116(11 Suppl):I89-97.

18. Nathan HJ, Rodriguez R, Wozny D, Dupuis JY, Rubens FD, Bryson GL, et al. Neuroprotective effect of mild hypothermia in patients undergoing coronary artery surgery with cardiopulmonary bypass: five-year follow-up of a randomized trial. J Thorac Cardiovasc Surg. 2007;133:1206-11.

19. Murkin JM, Newman SP, Stump DA, Blumenthal JA. Statement of consensus on assessment of neurobehavioral outcomes after cardiac surgery. Ann Thorac Surg. 1995;59:1289-95.

20. Turner-Bowker DMB, Bartley PJ, Ware JE Jr. SF-36 health survey \& "SF" bibliography. 3rd ed (1988-2000). Lincoln (RI): Quality Metric Incorporated; 2002

21. Ware J Jr, Kosinski M, Keller SD. A 12-item short-form health survey: construction of scales and preliminary tests of reliability and validity. Med Care. 1996;34:220-33.

22. Rasmussen LS, Larsen K, Houx P, Skovgaard LT, Hanning CD, Moller JT. The assessment of postoperative cognitive function. Acta Anaesth Scand. 2001;45:275-89.

23. Shroyer AL, Grover FL, Hattler B, Collins JF, McDonald GO, Kozora E, et al On-pump versus off-pump coronary-artery bypass surgery. $N$ Engl $\mathrm{J} \mathrm{Med}$. 2009;361:1827-37

24. Stroobant N, van Nooten G, De Bacquer D, Van Belleghem Y, Vingerhoets G Neuropsychological functioning 3-5 years after coronary artery bypass grafting: does the pump make a difference? Eur J Cardiothorac Surg. 2008;34:396-401.

25. Selnes OA, Grega MA, Borowicz LM Jr, Royall RM, McKhann GM, Baumgartner WA. Cognitive changes with coronary artery disease: a prospective study of coronary artery bypass graft patients and nonsurgical controls. Ann Thorac Surg. 2003;75:1377-84; discussion 1384-6. 